\title{
Quantum effects on the maximum in density of water as described by the TIP4PQ/2005 model
}

\author{
E. G. Noya, ${ }^{1}$ C. Vega, ${ }^{1, a)}$ L. M. Sesé, ${ }^{2}$ and R. Ramírez ${ }^{3}$ \\ ${ }^{1}$ Dep. Química-Física, Fac. de Química, Universidad Complutense de Madrid, E-28040 Madrid, Spain \\ ${ }^{2}$ Dept. Ciencias y Técnicas Fisicoquímicas, Fac. Ciencias, UNED, Paseo Senda del Rey 9 , \\ 28040 Madrid, Spain \\ ${ }^{3}$ Instituto de Ciencia de Materiales, CSIC, Campus de Cantoblanco, 28049 Madrid, Spain
}

(Received 15 July 2009; accepted 9 September 2009; published online 30 September 2009)

\begin{abstract}
Path integral simulations have been performed to determine the temperature of the maximum in density of water of the rigid, nonpolarizable TIP4PQ/2005 model treating long range Coulombic forces with the reaction field method. A maximum in density is found at $280 \mathrm{~K}$, just $3 \mathrm{~K}$ above the experimental value. In tritiated water the maximum occurs at a temperature about $12 \mathrm{~K}$ higher than in water, in reasonable agreement with the experimental result. Contrary to the usual assumption that the maximum in classical water is about $14 \mathrm{~K}$ above that in water, we found that for TIP4PQ/2005 this maximum is about $30 \mathrm{~K}$ above. For rigid water models the internal energy and the temperature of maximum density do not follow a linear behavior when plotted as a function of the inverse of the hydrogen mass. In addition, it is shown that, when used with Ewald sums, the TIP4PQ/2005 reproduces quite nicely not only the maximum in density of water, but also the liquid densities, the structure of liquid water and the vaporization enthalpy. It was shown in a previous work that it also reproduces reasonably well the density and relative stabilities of ices. Therefore the TIP4PQ/2005 model, while still simple, allows one to analyze the interplay between quantum effects related to atomic masses and intermolecular forces in water. () 2009 American Institute of Physics.

[doi:10.1063/1.3239471]
\end{abstract}

\section{INTRODUCTION}

One of the fingerprint properties of water is the existence of a maximum in density as a function of temperature at constant pressure that occurs at about $4 \mathrm{~K}$ above melting at normal pressure. With the increase in computer power it has been possible to show that popular water models exhibit a maximum in density, although at temperatures well below the experimental value. ${ }^{1}$ In the last years, several water models [TIP5P, ${ }^{2}$ TIP4P/2005 (Ref. 3)] that reproduce the experimental value of the temperature of maximum density (TMD) using classical simulations have been proposed.

The maximum in density for water $\left(\mathrm{H}_{2} \mathrm{O}\right)$, heavy water $\left({ }^{2} \mathrm{H}_{2} \mathrm{O}\right)$ and tritiated water $\left({ }^{3} \mathrm{H}_{2} \mathrm{O}\right)$ occurs at $3.98,11.18$, and $13.40{ }^{\circ} \mathrm{C}$, respectively. This shift in the TMD must be due to the existence of quantum effects related to the atomic masses and cannot be described by classical simulations (which would predict the same value of the TMD regardless of the molecular mass). Path integrals (PIs) can be used to simulate quantum effects in water. ${ }^{2,4-9}$ It seems now of interest to study in more detail the atomic mass quantum effect on the TMD. A crucial issue is the choice of the water model. The models developed for classical simulations incorporate implicitly quantum effects through the fit of the effective potential parameters to experimental data. In general if PI simulations are performed with a good classical model the agreement with experiment will deteriorate. ${ }^{10,11}$ Since good water models usually reproduce reasonably well the TMD of

${ }^{a)}$ Electronic mail: cvega@quim.ucm.es. water it seems important at this point to have water models able to reproduce the TMD of water within PI simulations. In fact recently Fanourgakis and Xanteas ${ }^{12}$ have proposed a flexible polarizable model (TTM2.1-F) that presents a density maximum ${ }^{8}$ at about $273 \mathrm{~K}$ when quantum effects are included (although the liquid densities are about $4 \%$ higher than in experiment). Mahoney and Jorgensen ${ }^{2}$ proposed a quantum version of the rigid nonpolarizable model TIP5P, denoted as TIP5P(PIMC), which exhibits a density maximum at about $273 \mathrm{~K}$. Habershon et al. ${ }^{13}$ have recently proposed a promising flexible nonpolarizable version of the TIP4P/2005 model, which has been denoted as q-TIP4P/F, with a density maximum at $279 \mathrm{~K}$ and providing good liquid densities when including quantum effects. Also by studying the properties of ices we have proposed ${ }^{14}$ a new version of TIP4P/2005, denoted as TIP4PQ/2005, that reproduces nicely the properties of the solid phases of water (within PI simulations). Although the model was developed for ices it would be of interest to check if this model is able to reproduce the TMD of real water.

A second issue of interest is to analyze in detail if PI simulations are able to predict the increase in the TMD with the molecular mass found experimentally. That would help to establish approximately the location of the TMD of water when treated classically (that would also correspond to the TMD of infinitely heavy water). By using the experimental values of the TMD for $\mathrm{H}_{2} \mathrm{O},{ }^{2} \mathrm{H}_{2} \mathrm{O}$, and ${ }^{3} \mathrm{H}_{2} \mathrm{O}$ Stillinger and Rahman $^{15}$ and Guillot ${ }^{16}$ already speculated that the TMD in the classical limit (i.e., extrapolating to infinite hydrogen mass) should be about $14 \mathrm{~K}$ higher than in real water. To 
estimate the value of the classical limit of the TMD for water it was assumed that this temperature is a linear function of the inverse of the mass of the hydrogen isotope.

In this work water will be described using the TIP4PQ/ 2005 model potential. This model is essentially identical to TIP4P/2005 except for the fact that the charge has been increased from the original value of $0.5564 e$ to $0.5764 e$. With this simple modification the model is able to reproduce the $g_{\text {oo }}$ of ice Ih and the densities and relative energies of the different ices. ${ }^{14}$ Such an increase in the proton charge has already been suggested elsewhere. ${ }^{2,7}$ Habershon et al. ${ }^{13}$ did not modify the charges of the original TIP4P/2005 but effectively increased the dipole moment of the model by introducing flexibility. Thus it seems that in all these cases the increase in the dipole moment of the molecule compensates for the introduction of nuclear quantum effects. Since TIP4P/ 2005 is a respectable model for classical simulations, ${ }^{17}$ it is of interest to explore the performance of their quantum counterparts.

\section{METHODOLY}

Quantum effects on the molecular orientation will be incorporated by using a method developed by Müser and co-workers ${ }^{18,19}$ to perform PI simulations of rigid bodies. The method has been described in detail elsewhere. The present calculations do not include intramolecular nuclear exchange effects, since those are only expected to be relevant at very low temperatures. The use of a rigid model will allow us to reduce the number of replicas (beads) used in the discretization of the PI from about $P=32$ (flexible model) ${ }^{7,20}$ to about $P=5$ (rigid model). ${ }^{2,6,21}$ This is due to the fact that a larger value of $P$ is required to describe the intramolecular vibration. Since in general the evaluation of the TMD requires extremely long runs, reducing the value of $P$ will reduce significantly the computer time.

The simulation box contained 360 water molecules in the classical simulations and 300 in the PI Monte Carlo (PIMC) simulations. Five or seven beads $(P=5$ or $P=7)$ were used to describe water within the PIMC simulations. We have indeed found that using $P=5(7)$ the energies are always within $3 \%(2 \%)$ of the value predicted for much larger values of $P$. The potential was truncated at $8.5 \AA$. Standard long range corrections [i.e., $g(r)=1$ ] have been added to the Lennard-Jones contribution, and the Coulombic interactions are corrected using the reaction field (RF) method, which has been shown to provide reasonable results for liquid water. ${ }^{22,23}$ Reaction field reduces the computational cost by a factor of about 3 compared to the use of Ewald sums. Finally, we have also computed the TMD using Ewald sums, as this is the approach that has been used in the fit of the model. The density for 6-7 temperatures along the isobar $p=1$ bar was estimated by performing simulations of around 4 million MC cycles each.

\section{RESULTS AND DISCUSSION}

The temperatures at the maximum in density along the isobar $p=1$ bar for $\mathrm{H}_{2} \mathrm{O}$, and classical TIP4PQ/2005 water are given in Table I and the results are presented graphically
TABLE I. TMD at $p=1$ bar as obtained from PIMC simulations for water (using $P=5$ and $P=7$ replicas), tritiated water $(P=5)$ and classical water with the TIP4PQ/2005 model.

\begin{tabular}{lcc}
\hline & \multicolumn{2}{c}{ TMD (K) } \\
\cline { 2 - 3 } System & TIP4PQ/2005 & Expt. \\
\hline Classical $\mathrm{H}_{2} \mathrm{O}(\mathrm{RF})$ & $307(2)$ & - \\
${ }^{3} \mathrm{H}_{2} \mathrm{O}(P=5)(\mathrm{RF})$ & $296(3)$ & 286.55 \\
$\mathrm{H}_{2} \mathrm{O}(P=5)(\mathrm{RF})$ & $284(2)$ & 277.13 \\
$\mathrm{H}_{2} \mathrm{O}(P=7)(\mathrm{RF})$ & $280(2)$ & 277.13 \\
$\mathrm{Classical}_{2} \mathrm{O}($ Ewald$)$ & $310(2)$ & - \\
$\mathrm{H}_{2} \mathrm{O}(P=5)($ Ewald $)$ & $285(2)$ & 277.13 \\
$\mathrm{H}_{2} \mathrm{O}(P=7)($ Ewald$)$ & $282(2)$ & 277.13 \\
\hline \hline
\end{tabular}

in Fig. 1(a). The TMDs have been located by fitting the densities in the neighborhood of the maximum to a cubic polynomial function. The TMD of quantum $\mathrm{H}_{2} \mathrm{O}$ (with the TIP4PQ/2005) is located at 284(2) $\mathrm{K}$ when using $P=5$ and 280(2) $\mathrm{K}$ when using $P=7$. Although the two results are quite close it seems that the temperature of the maximum moves to slightly lower temperatures as the number of beads is increased, and it could eventually get closer to the experimental value by further increasing the number of beads. The TMD of the classical model (using RF) is located at 307(2) K. Thus quantum effects shift the TMD of water by about 23(4) $\mathrm{K}$ if the results with $P=5$ are considered, or by about $27(4) \mathrm{K}$ if the results for $P=7$ are considered. Mahoney and Jorgensen ${ }^{2}$ found a similar shift for the TIP5P model. It is clear that for rigid models of water the difference
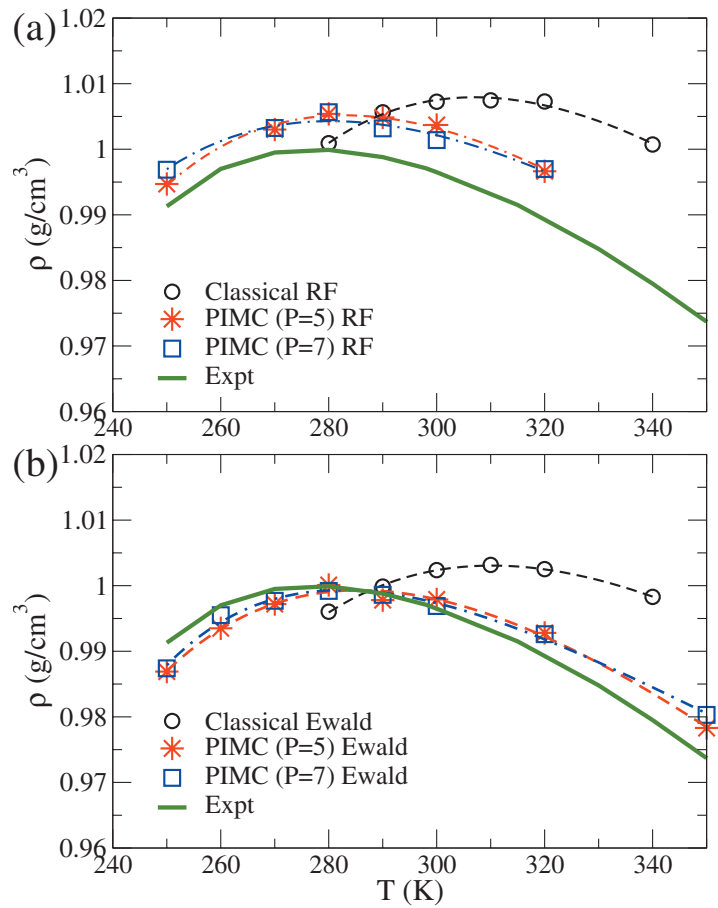

FIG. 1. Density along the isobar $p=1$ bar for water as obtained from PIMC simulations with $P=5$ and $P=7$ replicas and for classical water with the TIP4PQ/2005 model, treating the Coulombic forces with (a) RF, and (b) Ewald sums. The error in the density is about $0.001 \mathrm{~g} / \mathrm{cm}^{3}$. The dashed and dotted lines are fits to the results of the simulations. Experimental results are also shown. 


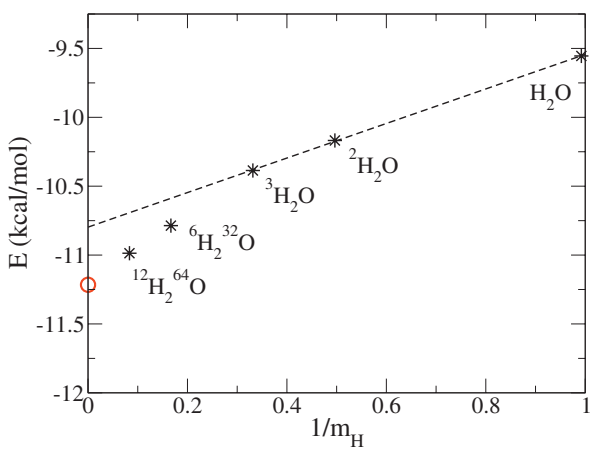

FIG. 2. Total energy for liquid water and isotopically substituted water at $T=298 \mathrm{~K}$ as a function of the inverse of hydrogen mass, as obtained from NVT PIMC simulations treating the Coulombic forces with Ewald sums. The selected number density is that of real water at room $T$ and $p$ (which yields a density of $\rho=0.9970 \mathrm{~g} / \mathrm{cm}^{3}$ for water). We used $P=10$ for $\mathrm{H}_{2} \mathrm{O}$, $P=7$ for ${ }^{2} \mathrm{H}_{2} \mathrm{O}$ and ${ }^{3} \mathrm{H}_{2} \mathrm{O}$, and $P=4$ for ${ }^{6} \mathrm{H}_{2}{ }^{32} \mathrm{O}$ and ${ }^{12} \mathrm{H}_{2}{ }^{64} \mathrm{O}$. The red circle shows the results of classical simulations. The straight line is a fit to the data for $\mathrm{H}_{2} \mathrm{O},{ }^{2} \mathrm{H}_{2} \mathrm{O}$, and ${ }^{3} \mathrm{H}_{2} \mathrm{O}$.

between the TMD of water and classical water is of about $30 \mathrm{~K}$. This shift differs from the estimate of $14 \mathrm{~K}$ previously suggested. ${ }^{16,24}$

In order to investigate the variation of quantum effects with the mass of the molecule (specially that of the hydrogen atoms) we performed NVT PIMC simulations at room temperature with the TIP4PQ/2005 model for several liquids of isotopically substituted water, including real species, such as $\mathrm{H}_{2} \mathrm{O},{ }^{2} \mathrm{H}_{2} \mathrm{O}$, and ${ }^{3} \mathrm{H}_{2} \mathrm{O}$, and some imaginary species, such as ${ }^{6} \mathrm{H}_{2}{ }^{32} \mathrm{O}$ and ${ }^{12} \mathrm{H}_{2}{ }^{64} \mathrm{O}$. The variation in the total energy with the inverse of hydrogen mass is shown in Fig. 2. It can be seen that the dependence on the total energy is not linear. Besides, a linear extrapolation of the data of $\mathrm{H}_{2} \mathrm{O},{ }^{2} \mathrm{H}_{2} \mathrm{O}$, and ${ }^{3} \mathrm{H}_{2} \mathrm{O}$ as a function of the inverse mass would also lead to a value in the classical limit that differs by about $4 \%$ of the true one. Thus for a rigid model of water the energy does not follow a linear behavior when plotted as a function of the inverse of the hydrogen mass. It is likely that the same applies to other thermodynamic functions. It seems reasonable to think that nuclear quantum effects on the TMD of water may be larger than expected, and that the previously estimated $14 \mathrm{~K}$ shift may be somewhat low.

What is the value of the TMD of classical water? This question cannot be answered from experiment since isotopes of hydrogen heavier than tritium are not available. It could be answered by computer simulation combining PI simulations and the exact value of the potential energy surface. Unfortunately such a surface is not available yet so that all descriptions of the potential energy surface are only approximate. That may explain why the melting temperature of ice Ih found from DFT simulations is of about $415 \mathrm{~K},{ }^{25}$ why the melting point of the potential TTM2.1-F is of about $228 \mathrm{~K}$, ${ }^{26}$ and why the melting point of the q-TIP4P/2005 is of about $251 \mathrm{~K}$ (Ref. 13) (the melting point of the TIP4PQ/2005 is probably close to that found by Habershon et al. for $\mathrm{q}-\mathrm{TIP} 4 \mathrm{P} / \mathrm{F})$. It is somewhat frustrating that three models which are able to reproduce the location of the TMD of water (i.e., TTM2.1-F, q-TIP4P/F and TIP4PQ/2005) fail to reproduce the melting point of ice, predicting differences between the TMD and the melting point temperatures of about
$42 \mathrm{~K}$ (Ref. 26) for the TTM2.1-F and of about $28 \mathrm{~K}$ (Ref. 13) for the q-TIP4P/F, which should be compared to the experimental value of the shift, $4 \mathrm{~K}$. This drawback is also manifested in classical simulations with rigid models, for which a difference of about 21-37 K was found. ${ }^{1,27}$ Since all these models are approximate it is not obvious which one would yield better estimates of the TMD of classical water. In fact for the TTM2.1-F (a flexible polarizable model) the temperature of the TMD is predicted to be roughly the same for water and classical water, ${ }^{8}$ a surprising result when one considers that experimentally the TMD of ${ }^{3} \mathrm{H}_{2} \mathrm{O}$ is $9 \mathrm{~K}$ higher than that of water. We have computed the TMD for tritiated water $\left({ }^{3} \mathrm{H}_{2} \mathrm{O}\right)$ using the TIP4PQ/2005 model and five replicas $(P=5)$. We obtained that the TMD is located at $296 \mathrm{~K}$, a temperature $12 \mathrm{~K}$ above that of water (using the same number of beads). The trend is predicted correctly although the magnitude is overestimated. It is likely that the most accurate estimate of the TMD of classical water would be that obtained from a model predicting a shift of $9 \mathrm{~K}$ (the experimental value) in the TMD of ${ }^{3} \mathrm{H}_{2} \mathrm{O}$ with respect to $\mathrm{H}_{2} \mathrm{O}$. In any case it is clear that the simple rigid model used here (which cannot describe the deformation of the molecular geometry with the hydrogen isotope suggested by the experiments ${ }^{28}$ ) does at least a reasonably qualitative job in describing the experimental shift of the TMD from $\mathrm{H}_{2} \mathrm{O}$ to ${ }^{3} \mathrm{H}_{2} \mathrm{O}$.

Although RF reduces considerably the computer time it would be of interest to determine the TMD of the TIP4PQ/ 2005 by using Ewald sums. Note that the TIP4PQ/2005 was parameterized using Ewald sums for the solid phases of water. ${ }^{14}$ The calculated densities and energies along the isobar $p=1$ bar using Ewald sums for $P=5$ and $P=7$ are given in Table II and the corresponding TMDs are given in Table I. In Fig. 1(b) these data are shown together with the experimental results. By comparing the results of quantum $(P=7)$ and classical simulations, it is observed that quantum effects (in the total energy $E$ ) amount to about $1.8 \mathrm{kcal} / \mathrm{mol}$ in this range of temperatures. The main contributions to the quantum effects come from the rotational kinetic energy (about $0.6 \mathrm{kcal} / \mathrm{mol}$ ) and from the potential energy (about $1.2 \mathrm{kcal} /$ mol). Quantum effects over the translational kinetic energy are much less important. By comparing with the data of Fig. 1(a) it is seen that densities obtained by using Ewald sums are about $0.006 \mathrm{~g} / \mathrm{cm}^{3}$ lower than those obtained from the $\mathrm{RF}$ technique and closer to the experimental results. The TMD is located at $285(2) \mathrm{K}$ when using $P=5$ replicas and at 282(2) K when using $P=7$. Similarly to what was found with $\mathrm{RF}$, the increase in the number of replicas seems to shift the TMD to slightly lower temperatures. In summary, the use of Ewald sums reduces the densities but does not modify much the location of the temperature of the TMD. The TIP4PQ/ 2005 model with Ewald sums yields a TMD located around 282(2) K, and with densities close to the experimental values (the deviation from experimental densities is smaller than $1 \%$ in the whole range of temperatures studied). By using Ewald sums we have computed the vaporization enthalpy and structure at room pressure and temperature. The vaporization enthalpy of the model was found to be of $10.56 \mathrm{kcal} /$ mol (with the self-energy correction proposed by Berendsen et $a{ }^{29}$ for nonpolarizable models), which is in very good 
TABLE II. Densities and contributions to the total energy $E$ as a function of temperature along the isobar $p$ $=1$ bar using PIMC simulations with $P=5$ and $P=7$ replicas for TIP4PQ/2005 water. The long range Coulombic interactions were treated using Ewald sums. $U$ stands for potential energy, and $K_{\text {tras }}$ and $K_{\text {rot }}$ for translational and rotational kinetic energy, respectively. Energies are given in $\mathrm{kcal} / \mathrm{mol}$ and densities in $\mathrm{g} / \mathrm{cm}^{3}$. The uncertainty is about $0.001 \mathrm{~g} / \mathrm{cm}^{3}$ in the density, $0.001 \mathrm{kcal} / \mathrm{mol}$ in the translational and rotational energies, and 0.01 $\mathrm{kcal} / \mathrm{mol}$ in the potential and total energies. For $P=5$ and $P=7$, the classical kinetic energy (translational or rotational), i.e., $3 / 2 R T$, is also given for comparison with the quantum value.

\begin{tabular}{cccccccc}
\hline \hline$T(\mathrm{~K})$ & $3 / 2 R T$ & $K_{\text {tras }}$ & $K_{\text {rot }}$ & $U$ & $E$ & $\rho$ & $\rho_{\text {exp }}$ \\
\hline$P=1$ & & & & & & & \\
280 & 0.834 & 0.834 & 0.834 & -13.297 & -11.629 & 0.9960 & 0.9999 \\
290 & 0.863 & 0.863 & 0.863 & -13.116 & -11.390 & 0.9999 & 0.9988 \\
300 & 0.893 & 0.893 & 0.893 & -12.939 & -11.153 & 1.0024 & 0.9965 \\
310 & 0.923 & 0.923 & 0.923 & -12.767 & -10.921 & 1.0032 & 0.9934 \\
320 & 0.953 & 0.953 & 0.953 & -12.600 & -10.694 & 1.0025 & 0.9894 \\
340 & 1.012 & 1.012 & 1.012 & -12.281 & -10.257 & 0.9983 & 0.9795 \\
& & & & & & & \\
$P=5$ & & & & & & & \\
250 & 0.744 & 0.824 & 1.345 & -12.662 & -10.492 & 0.9869 & 0.9913 \\
260 & 0.774 & 0.850 & 1.354 & -12.507 & -10.302 & 0.9935 & 0.9970 \\
270 & 0.804 & 0.877 & 1.364 & -12.352 & -10.111 & 0.9972 & 0.9995 \\
280 & 0.834 & 0.904 & 1.372 & -12.201 & -9.925 & 1.0001 & 0.9999 \\
290 & 0.863 & 0.930 & 1.383 & -12.067 & -9.754 & 0.9978 & 0.9988 \\
300 & 0.893 & 0.957 & 1.395 & -11.923 & -9.571 & 0.9979 & 0.9965 \\
320 & 0.953 & 1.011 & 1.420 & -11.656 & -9.224 & 0.9928 & 0.9894 \\
& & & & & & & \\
$P=7$ & & & & & & & \\
250 & 0.744 & 0.825 & 1.387 & -12.599 & -10.386 & 0.9874 & 0.9913 \\
260 & 0.774 & 0.853 & 1.392 & -12.442 & -10.197 & 0.9955 & 0.9970 \\
270 & 0.804 & 0.878 & 1.397 & -12.290 & -10.015 & 0.9977 & 0.9995 \\
280 & 0.834 & 0.905 & 1.406 & -12.152 & -9.842 & 0.9992 & 0.9999 \\
290 & 0.863 & 0.931 & 1.414 & -12.013 & -9.667 & 0.9986 & 0.9988 \\
300 & 0.893 & 0.958 & 1.425 & -11.876 & -9.493 & 0.9969 & 0.9965 \\
320 & 0.953 & 1.012 & 1.445 & -11.615 & -9.157 & 0.9926 & 0.9894 \\
\hline \hline & & & & & & & \\
& & & & & & & \\
& & & & & & & \\
\end{tabular}

agreement with the experimental value $(10.52 \mathrm{kcal} / \mathrm{mol})$. From the data given in Table II, it is also possible to compute the heat capacity at constant pressure by simply fitting the enthalpy $(H=E+p V)$ to a quadratic polynomial function, and taking the derivative of the enthalpy with respect to the temperature. PIMC simulations with the TIP4PQ/2005 model $(P=7)$ predict a heat capacity of $17.7 \mathrm{cal} \mathrm{mol}^{-1} \mathrm{~K}^{-1}$ at $280 \mathrm{~K}$, which compares quite well with the experimental result, $17.9 \mathrm{cal} \mathrm{mol}^{-1} \mathrm{~K}^{-1}$. This supposes an improvement over classical simulation with the TIP4P/2005 model that give a heat capacity of about $21.5 \mathrm{cal} \mathrm{mol}^{-1} \mathrm{~K}^{-1}$ at this temperature.

In Fig. 3 the structure of the liquid is presented together with experimental data. As it can be seen the predictions of the TIP4PQ/2005 model agree reasonably well with the experimental values. ${ }^{30}$ In addition, we have also calculated the effect of hydrogen mass on the structure of the liquid (see Fig. 4). Quite recently, Soper and Benmore ${ }^{28}$ have measured the differences in the structure of water and deuterated water at room pressure and temperature, and these data can be used as a further test of our model. The results of the PIMC simulations agree qualitatively with experimental results. In agreement with the experiments, the peaks in the oxygenoxygen distribution function are lower and broader in water than in deuterated water. Moreover we found that the first peak in the hydrogen-hydrogen function is located at slightly shorter distances in deuterated water than in water, i.e., the same trend found in experiments. Note, however, that it is likely that a quantitative agreement with experiments cannot be obtained with a rigid model with the same geometry for water and deuterated water, as it has been found experimentally that the geometry of water changes somewhat upon isotopic substitution of hydrogen. ${ }^{28,31}$

Thus the TIP4PQ/2005 appears as a reasonable and computationally cheap model which allows one to study the in-

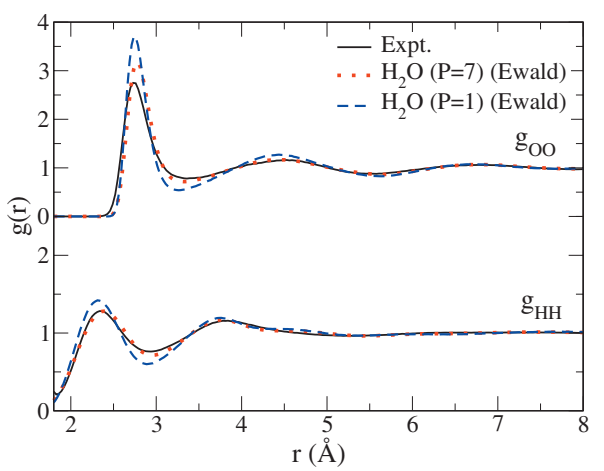

FIG. 3. Atomic distribution functions of liquid water at $T=298 \mathrm{~K}$ and $\rho$ $=0.9970 \mathrm{~g} / \mathrm{cm}^{3}$ as obtained from quantum $(P=7)$ and classical $(P=1)$ simulations with the TIP4PQ/2005 model. Experimental data (Ref. 30) are also shown. 


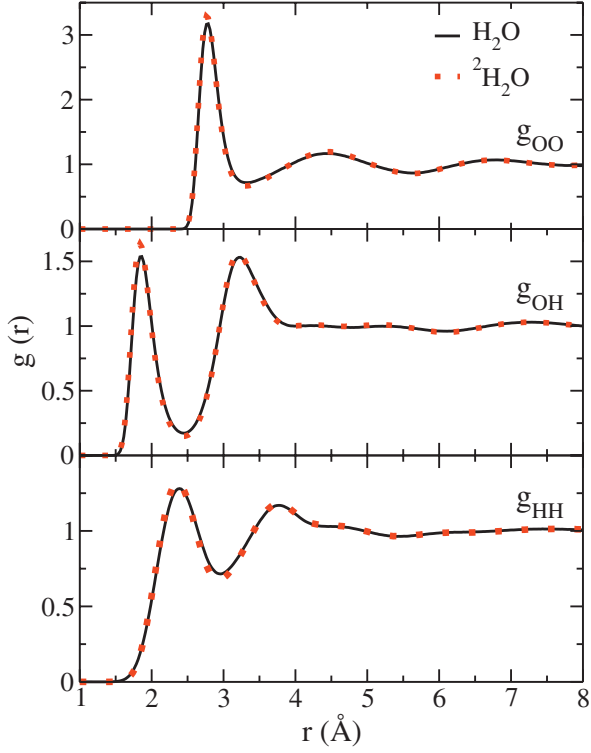

FIG. 4. Atomic distribution functions of liquid water and deuterated water at $T=298 \mathrm{~K}$ and $p=1$ bar as obtained from quantum $(P=7)$ simulations with the TIP4PQ/2005 model.

terplay between quantum effects related to atomic masses and intermolecular forces (which yield the librational bands located below $900 \mathrm{~cm}^{-1}$ in the IR spectrum). The TIP4PQ/ 2005 (with Ewald sums) reproduces reasonably well the properties of ices, ${ }^{14}$ the TMD of real water, the liquid densities, the structure and the vaporization enthalpy. The difference between the TMD of the quantum and classical simulations is of about $30 \mathrm{~K}$ for this model. This shift of $30 \mathrm{~K}$ is an upper limit for the difference between the TMDs in the quantum and classic limits of real water, as we have found that the model predicts an increase in the TMD when going from $\mathrm{H}_{2} \mathrm{O}$ to ${ }^{3} \mathrm{H}_{2} \mathrm{O}$, which, even though agrees qualitatively with the experimental trend, overestimates the magnitude of the shift. For rigid models the classical limit cannot be estimated by extrapolating linearly the results of light isotopes because the properties are not linear functions of the inverse of the hydrogen mass.

Obviously only flexible models can deal with the mass dependent quantum effects on the intramolecular degrees of freedom (stretching and bending which appear at frequencies above $1200 \mathrm{~cm}^{-1}$ in the IR spectrum). However, it should also be pointed out that since flexible models are also approximate they may yield quite different predictions for the behavior of classical water (as an example the ratio of the diffusion coefficient $D_{\text {quantum }} / D_{\text {classical }}$ has been predicted to be 1.4 and 1.1, respectively, using two different flexible models $\left.{ }^{13}\right)$. Nevertheless it is expected that in the future, the comparison between the results of TIP4P/2005, TIP4PQ/
2005 and q-TIP4P/F (which all belong to the TIP4P/2005 family) may help to clarify the importance of quantum effects $^{32}$ (both intermolecular and intramolecular) on the properties of water.

\section{ACKNOWLEDGMENTS}

This work has been funded by DGI (Spain) (Grant Nos. FIS2007-66079-C02-01, FIS2006-12117-C04-03), and by the Comunidad Autonoma de Madrid (Grant No. S-0505/ ESP/0299). E.G.N. thanks the award of a Juan de la Cierva fellowship. Helpful discussions with Professor J. L. F. Abascal and Dr. C. McBride are gratefully acknowledged. We thank Dr. Habershon and Professor D. E. Manolopoulos for sending us a preprint of their work previous to publication.

${ }^{1}$ C. Vega and J. L. F. Abascal, J. Chem. Phys. 123, 144504 (2005).

${ }^{2}$ M. Mahoney and W. L. Jorgensen, J. Chem. Phys. 115, 10758 (2001).

${ }^{3}$ J. L. F. Abascal and C. Vega, J. Chem. Phys. 123, 234505 (2005).

${ }^{4}$ D. Chandler and P. G. Wolynes, J. Chem. Phys. 74, 4078 (1981).

${ }^{5}$ R. A. Kuharski and P. J. Rossky, J. Chem. Phys. 82, 5164 (1985).

${ }^{6}$ L. H. de la Peña, M. S. Gulam Razul, and P. G. Kusalik, J. Chem. Phys. 123, 144506 (2005).

${ }^{7}$ F. Paesani, W. Zhang, D. A. Case, T. E. Cheatham III, and G. A. Voth, J. Chem. Phys. 125, 184507 (2006).

${ }^{8}$ F. Paesani, S. Iuchi, and G. A. Voth, J. Chem. Phys. 127, 074506 (2007).

${ }^{9}$ C. D. Wick and G. K. Schenter, J. Chem. Phys. 124, 114505 (2006).

${ }^{10}$ L. M. Sesé, J. Chem. Phys. 126, 164508 (2007).

${ }^{11}$ R. Ramírez, C. P. Herrero, A. Antonelli, and E. R. Hernández, J. Chem. Phys. 129, 064110 (2008).

${ }^{12}$ G. S. Fanourgakis and S. S. Xanteas, J. Phys. Chem. A 110, 4100 (2006).

${ }^{13} \mathrm{~S}$. Habershon, T. E. Markland, and D. E. Manolopoulos, J. Chem. Phys. 131, 024501 (2009).

${ }^{14}$ C. McBride, C. Vega, E. G. Noya, R. Ramírez, and L. M. Sesé, J. Chem. Phys. 131, 024506 (2009).

${ }^{15}$ F. H. Stillinger and A. Rahman, J. Chem. Phys. 60, 1545 (1974).

${ }^{16}$ B. Guillot, J. Mol. Liq. 101, 219 (2002).

${ }^{17}$ C. Vega, J. L. F. Abascal, M. M. Conde, and J. L. Aragones, Faraday Discuss. 141, 251 (2009).

${ }^{18}$ D. Marx and M. H. Müser, J. Phys.: Condens. Matter 11, R117 (1999).

${ }^{19}$ M. H. Müser and B. J. Berne, Phys. Rev. Lett. 77, 2638 (1996).

${ }^{20}$ W. Shinoda and M. Shiga, Phys. Rev. E 71, 041204 (2005).

${ }^{21}$ L. H. de la Peña and P. G. Kusalik, J. Chem. Phys. 121, 5992 (2004).

${ }^{22}$ M. Lisal, J. Kolafa, and I. Nezbeda, J. Chem. Phys. 117, 8892 (2004).

${ }^{23}$ B. Garzon, S. Lago, and C. Vega, Chem. Phys. Lett. 231, 366 (1994).

${ }^{24}$ S. R. Billeter, P. M. King, and W. F. van Gunsteren, J. Chem. Phys. 100, 6692 (1994)

${ }^{25}$ S. Yoo, X. C. Zeng, and S. S. Xanteas, J. Chem. Phys. 130, 221102 (2009).

${ }^{26}$ F. Paesani and G. A. Voth, J. Phys. Chem. C 112, 324 (2008).

${ }^{27}$ C. Vega, E. Sanz, and J. L. F. Abascal, J. Chem. Phys. 122, 114507 (2005).

${ }^{28}$ A. K. Soper and C. J. Benmore, Phys. Rev. Lett. 101, 065502 (2008).

${ }^{29}$ H. J. C. Berendsen, J. R. Grigera, and T. P. Straatsma, J. Phys. Chem. 91, 6269 (1987).

${ }^{30}$ A. K. Soper, Chem. Phys. 258, 121 (2000).

${ }^{31}$ B. Tomberli, C. J. Benmore, P. A. Egelstaff, J. Neuefeind, and V. Honkimaki, J. Phys.: Condens. Matter 12, 2597 (2000).

${ }^{32}$ M. J. Gillan, The Path-Integral Simulation of Quantum Systems, Nato ASI Series C Vol. 293 (Kluwer, Dordrecht, 1990), Chap. 6, pp. 155-188. 